Review

\title{
Polysaccharide Nanosystems for Future Progress in Cardiovascular Pathologies
}

\author{
Amanda Karine Andriola Silva, Didier Letourneur, Cédric Chauvierre ${ }^{凶}$ \\ Inserm, U698, Cardiovascular Bio-Engineering; X. Bichat hospital, 46 rue H. Huchard, F-75018, Paris, France; Université Paris 13, Sorbonne \\ Paris Cité, F-93430, Villetaneuse, France. \\ $\square$ Corresponding author: Inserm, U698, Cardiovascular Bio-Engineering; X. Bichat hospital, 46 rue H. Huchard, F-75018, Paris, France; Tel: \\ (33) 14025 8600; Fax: (33) 140258602 E-mail address: cedric.chauvierre@inserm.fr. \\ (c) Ivyspring International Publisher. This is an open-access article distributed under the terms of the Creative Commons License (http://creativecommons.org/ \\ licenses/by-nc-nd/3.0/). Reproduction is permitted for personal, noncommercial use, provided that the article is in whole, unmodified, and properly cited.
}

Received: 2013.09.17; Accepted: 2013.11.16; Published: 2014.03.1 I

\begin{abstract}
Natural polysaccharides have received a lot of attention in the biomedical field. Indeed, sources of polysaccharides, extracted or produced from plants, bacteria, fungi or algae, are diverse and renewable. Moreover, recent progresses in polysaccharide chemistry and nanotechnologies allow elaborating new dedicated nanosystems. Polysaccharide-based nanosystems may be designed for interacting in several biological processes. In particular, the atherothrombotic pathology is highly concerned by polysaccharide-mediated recognition. Atherothrombotic diseases, regardless of the anatomical localization, remain the main causes of morbidity and mortality in the industrialized world. This review intends to provide an overview on polysaccharide-based nanosystems as drug delivery systems and targeted contrast agents for molecular imaging with an emphasis on the treatment and imaging of cardiovascular pathologies.
\end{abstract}

Key words: Polysaccharides; Nanosystems; Atherothrombosis; Drug delivery; Molecular imaging.

\section{Introduction}

Polysaccharides consist of long carbohydrate molecules containing repeated monosaccharide units which are joined together by means of glycosidic bonds. Polysaccharides represent the most abundant biomolecules in nature with essential roles in a wide range of processes in living systems.[1-3] Polysaccharides are highly biocompatible and biodegradable. They can be classified by their origin: vegetal origin (e.g. pectin), algal origin (e.g. alginate), microbial origin (e.g. dextran, xanthan gum), and animal origin (chitosan, heparin).[4] Polysaccharides may also be classified by their charge: cationic (chitosan), anionic (hyaluronic acid, heparin) and nonionic (dextran). Most natural polysaccharides display hydrophilic groups such as hydroxyl, carboxyl and amino groups.[5] Due to the presence of various functional groups on molecular chains, polysaccharides may be easily chemically modified.
The chemical diversity of polysaccharides contributes to their variety in functions. Besides their uses as energy sources, such natural polymers are endowed with important structural functions since they are important components in biological systems as primary constituents of eukaryotic cell surface and the extracellular environment.[6] Additionally, they mediate important recognition events in biological process through interactions with proteins and other biological entities.[6, 7]

A drawback associated to polysaccharides is related to their natural variability and difficult laboratorial synthesis. These disadvantages are also shared with other natural polymers such as proteins, which additionally may also elicit immunogenic responses. In contrast, synthetic polymers may be synthesized in laboratory with high batch-to-batch uniformity, predictable properties and tailored structure for opti- 
mized features.[8] The main disadvantage of most of synthetic polymers is their low biodegradability. However, some synthetic polymers are known to degrade in vivo, most belonging to polyester family, which includes polyglycolides and polylactides. Even though, they still present poor biocompatibility, loss of mechanical properties during degradation and release of acidic degradation products.[9]

Knowledge at the cellular and molecular levels has increased greatly. Therefore, tremendous advances have been made in defining the appropriate polysaccharide involved in a precise biological process.[10] The atherothrombotic pathology is highly concerned by polysaccharide-mediated recognition both enabling targeting and inhibition of biomolecules. In order to gain insight on polysaccharide interaction in the context of atherothrombotic diseases and appraise structure-function relationships, it is valuable to highlight some key processes involved in polysaccharide recognition. The following section deals with the advantages of associating nanosystems to polysaccharides, conjugation methods are introduced and characterization techniques are summarized. Afterwards, polysaccharide nanosystems based on sialyl Lewis $x$, heparin, hyaluronic acid, fucoidan, chitosan, cylcodextrin and dextran are individually overviewed considering their application on the treatment and imaging of atherothrombotic disease. The final section includes concluding remarks and perspectives.

\section{Structure-function of polysaccharides and features of nanoparticles for athero- thrombosis management}

\section{Polysaccharide-mediated recognition in atherothrombotic process}

The structural diversity of polysaccharides confers them different binding affinities that directly relates to their role in several important physiological and pathological processes. Polysaccharides are involved in a wide variety of physiological events such as cell signaling and adhesion. Polysaccharide binding to proteins and signaling molecules modulate their activity, thus influencing fundamental biological processes.[6] For instance, heparan sulfate chains of proteoglycans enable interaction with molecules such as fibronectin, laminin and collagen, which is important for the organization of basement membrane and extracellular matrix as well as cell adhesion to matrix.[11] Furthermore, heparan sulfate and other related polysaccharides such as fucoidan and heparin bind sugar recognition domains on selectins, mediating the interaction with activated platelets and endothelial cells $[12,13]$ by mimicking the binding of the oligosaccharide sialyl Lewis ${ }^{\mathrm{x}}$ with $\mathrm{E}, \mathrm{L}$ and $\mathrm{P}$ selectins (Fig. 1).

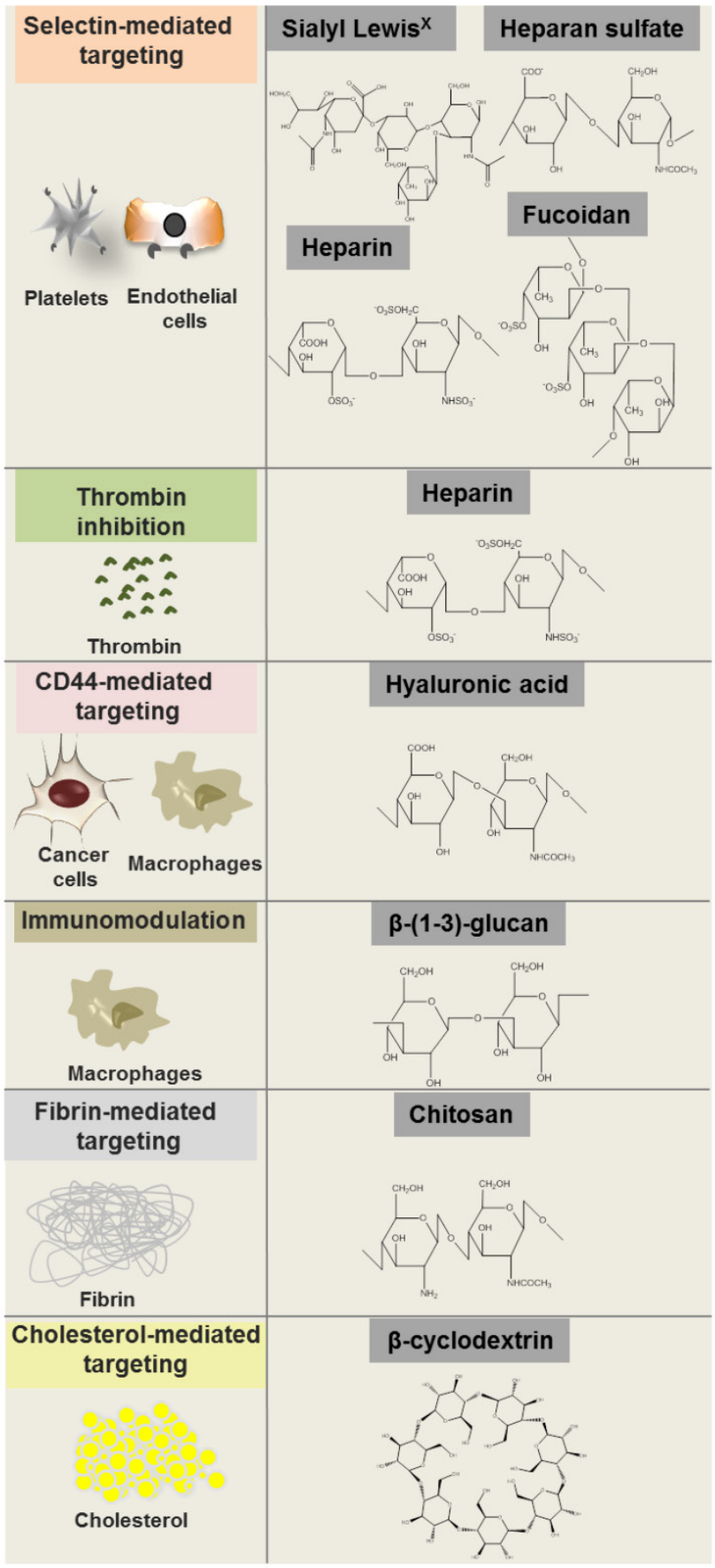

Figure I: Polysaccharide molecular structures and some of their biological targets.

Polysaccharides also participate in pathological processes. Many bacterial pathogens may initiate infections through the binding of adhesins to mammalian cell surface compounds, including proteins, glycolipids, and polysaccharides.[14] Bacterial exopolysaccharides participate in both cell-cell recognition and interaction as well as in the formation of a physical protective barrier protecting them from antibody 
binding and phagocytosis.[15] As an additional example, polysaccharides such as hyaluronic acid are known to interact with macrophages.

Polysaccharides may also be used as therapeutic agents. Certain polysaccharides are immunomodulators, affecting the regulation of immune responses during the progression of infectious diseases via interactions with $\mathrm{T}$ cells, monocytes, macrophages, and polymorphonuclear lymphocytes.[17-19] This is the case of $\beta$-(1-3)-glucan. Its recognition and the subsequent receptor binding process via $\beta$-(1-3)-glucan binding proteins is the first step in mediating the activating effects.[20] The activation of signal transduction pathways enhance the antimicrobial activity of mononuclear cells and neutrophils while also stimulating the proliferation of monocytes and macrophages.[17] In a clinical study, the immunomodulatory properties of $\beta$-(1-3)-glucan were found to enhance ulcer healing.[21]

Another remarkable example of a polysaccharide as a therapeutic agent is heparin due to its interaction with antithrombin, which is an important player in coagulation process. At concentrations present in blood, antithrombin slowly inhibits blood clotting because it exists in a low reactivity state. When heparin binds to antithrombin, it induces a conformational change in the molecule which results in a greatly accelerated reaction with thrombin, enabling fast thrombin inactivation.[22, 23] Binding of heparin to antithrombin is enhanced by the distinct sequence of five saccharides.[24] This region is composed of one glucuronic acid unit, one a-L-iduronic acid unit and three glucosamine units, two of which are invariably $\mathrm{N}$-sulphated whereas the remaining one may be either $\mathrm{N}$-acetylated or $\mathrm{N}$-sulphated.[25] Heparin is also well described to bind to vascular smooth muscle cells inhibiting their proliferation.[26]

Besides heparin, several polysaccharides are able to interact in cardiovascular diseases such as atherothrombosis. Cardiovascular diseases are predominant causes of death in developed countries. They also account for considerable morbidity and mortality worldwide, representing a substantial economic burden.[27, 28] Atherosclerosis is an important arterial wall disease characterized by focal lesions with asymmetric thickenings of the innermost layer of the artery that may lead to ischemia of the heart, brain, or extremities.[29] Dilatation or rupture is observed in the case of aneurysms. In such arterial disorders, macrophages, activated smooth muscle cells, lipids such as cholesterol, and extracellular matrix are major players.[30] Although advanced atherosclerotic lesions can lead to ischemic symptoms as a consequence of progressive narrowing of the vessel lumen, acute and severe cardiovascular events are generally in- duced by plaque rupture.[31] Plaque rupture is one of the leading causes of thrombosis. Thrombus is also observed in human abdominal aorta aneurysms.[32] Blood exposure to prothrombotic material from the core of the plaque disrupts homeostasis.[33] Platelets adhere to the sub-endothelial matrix and aggregate promoting clot formation and vascular occlusion, while concomitant blood coagulation culminates in the generation of thrombin and fibrin.[27, 34] Polysaccharides are able to recognize biological molecules involved in atherothrombotic process. Selectins, macrophage receptors, fibrin, antithrombin and also cholesterol represent some of the targets for polysaccharide interaction in atherothrombotic process, as summarized in Figure 1. Some specific polysaccharide interaction with each of these target molecules will be individually overviewed in the last section considering polysaccharide-based nanosystems and their application on the treatment and imaging of atherothrombotic disease. Before this, the advantages of associating polysaccharides to nanosystems will be discussed and methods for producing and characterizing polysaccharide-based nanosystems will be outlined.

\section{Structural features of nanosystems determining their fate and performance in atherothrombosis management}

Nanosystems are in the same scale length of biologic molecules. Therefore, there is a huge interest in exploiting them for the treatment and diagnosis of diseases at the molecular level.[35] In general, nanosystems present at least one dimension in the 1-100 nanometer scale. Indeed, nanosized carriers are small enough to interact with receptor targets while being large enough to transport drugs or imaging agents simultaneously avoiding renal clearance.[36, 37] Nanosystems above the renal clearance threshold circulate for a longer time,[38] which may influence their uptake in regions of leaky vasculature such as in atherosclerotic plaques. Plaques present a neovasculogenesis pattern similar to that observed in cancerous tumor growth, in which atypical blood vessels both defective and immature are formed. This implies in changes of the dynamics of macromolecular transport known as the enhanced permeability and retention (EPR) effect, allowing macromolecules or nanoassemblies to pass into the interstitial tissue[39, 40] (Fig. 2).

By virtue of their reduced size, nanosystems also have high surface area to volume ratios. This implies an abundant surface area available to ligand decoration either for targeting and drug/probe coupling.[35, 41, 42] Additionally, a high internal volume is provided by nanosystems. Notably, the internal volume 
of spherical particles increases cubically as external surface area is squared. Such internal volume is highly valuable for encapsulating drugs or imaging agents.[42] Thereby, loading is enabled while simultaneous protection against chemical or enzymatic degradation is conferred. Besides, nanosystem encapsulation enable controlled release for tuning the delivery rate of the encapsulated agent.[43] These are important features of nanosystems that make them particularly well suited as drug delivery carriers for the management of cardiovascular diseases. For instance, sustained release of heparin is quite desired for reducing intimal hyperplasia after vascular interventions.[44] As an additional example, tissue-plasminogen activator was encapsulated into nanoparticles to provide protection against inactivation by against inhibitors in plasma.[45] Drug protection and sustained release conferred by nanoparticles may remarkably contribute to a higher efficiency in the treatment of cardiovascular diseases.

Notably, nanosystems have improved optical, electronic and magnetic properties that markedly differ from the properties of their atoms and macroscopic material counterparts, which is a direct consequence of the behavior of electrons in the nanomet- ric confinement.[35] This particularly applies to cadmium selenide semiconductor nanostructures and iron oxide nanoparticles whose optical and magnetic properties, respectively, are strongly dictated by size.[46, 47]

Another important feature of nanosystems is their rapid recognition by the reticuloendothelial system (RES). They are rapidly cleared from the bloodstream upon intravenous injection, as a function of their size and surface characteristics.[48] The rapid uptake of nanosystems is quite undesirable if long circulation time is required. In this case, coating approaches should be implemented in order to confer stealthness to nanosystems, as it will be discussed ahead. Otherwise, the spontaneous RES uptake of nanosystems may also be of interest, considering macrophage central role in inflammatory diseases, such as in atherothrombotic disease.[49] The advantages related to the physical properties and biological features of nanosystems are briefly depicted in Figure 2.

Associating nanosystems and polysaccharide implies in benefiting from all the advantages provided by nanosystems while taking profit from recognition properties from polysaccharides.

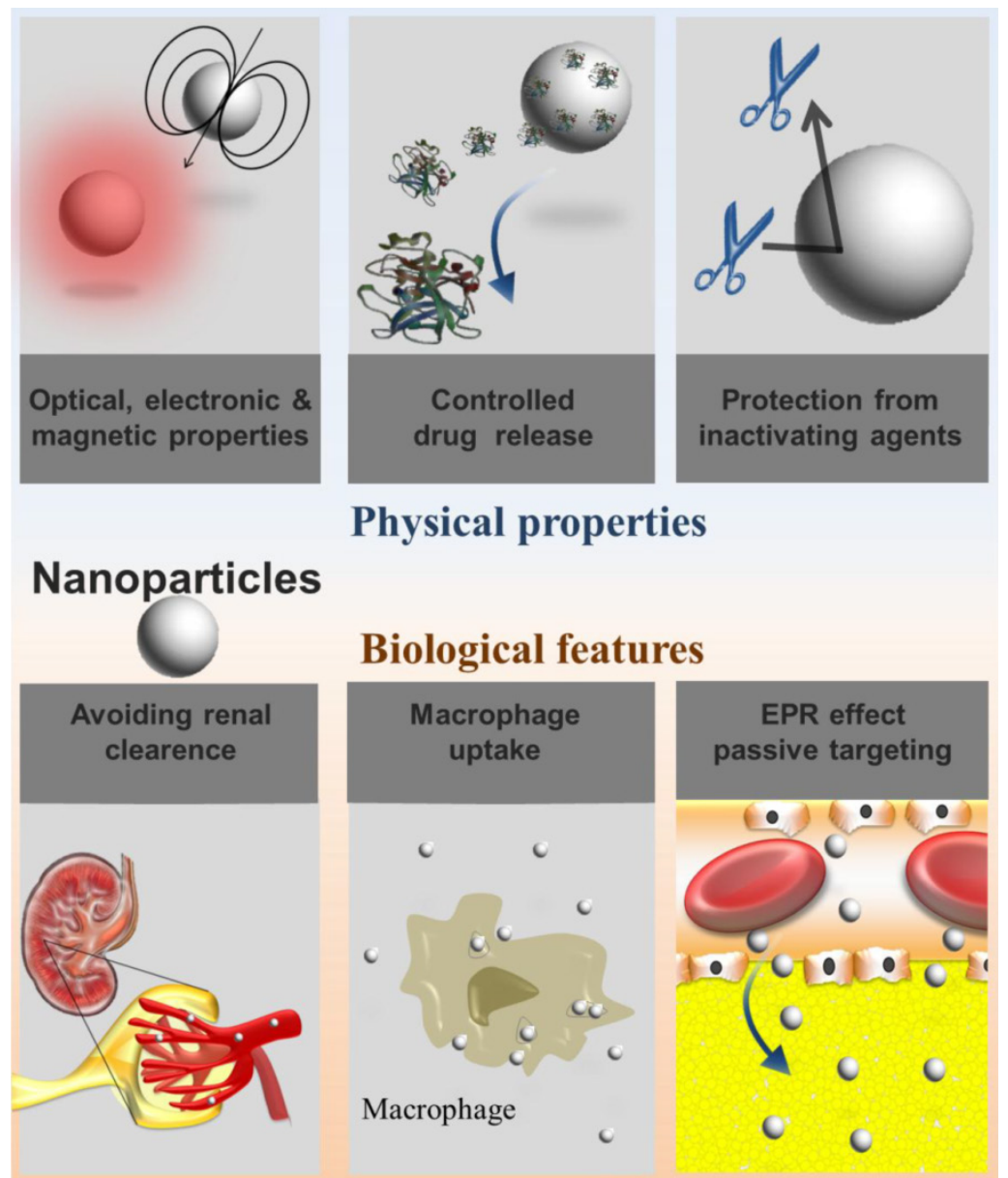

Figure 2: Physical properties and biological features of nanosystems considering their applications as drug carriers or imaging agents. 


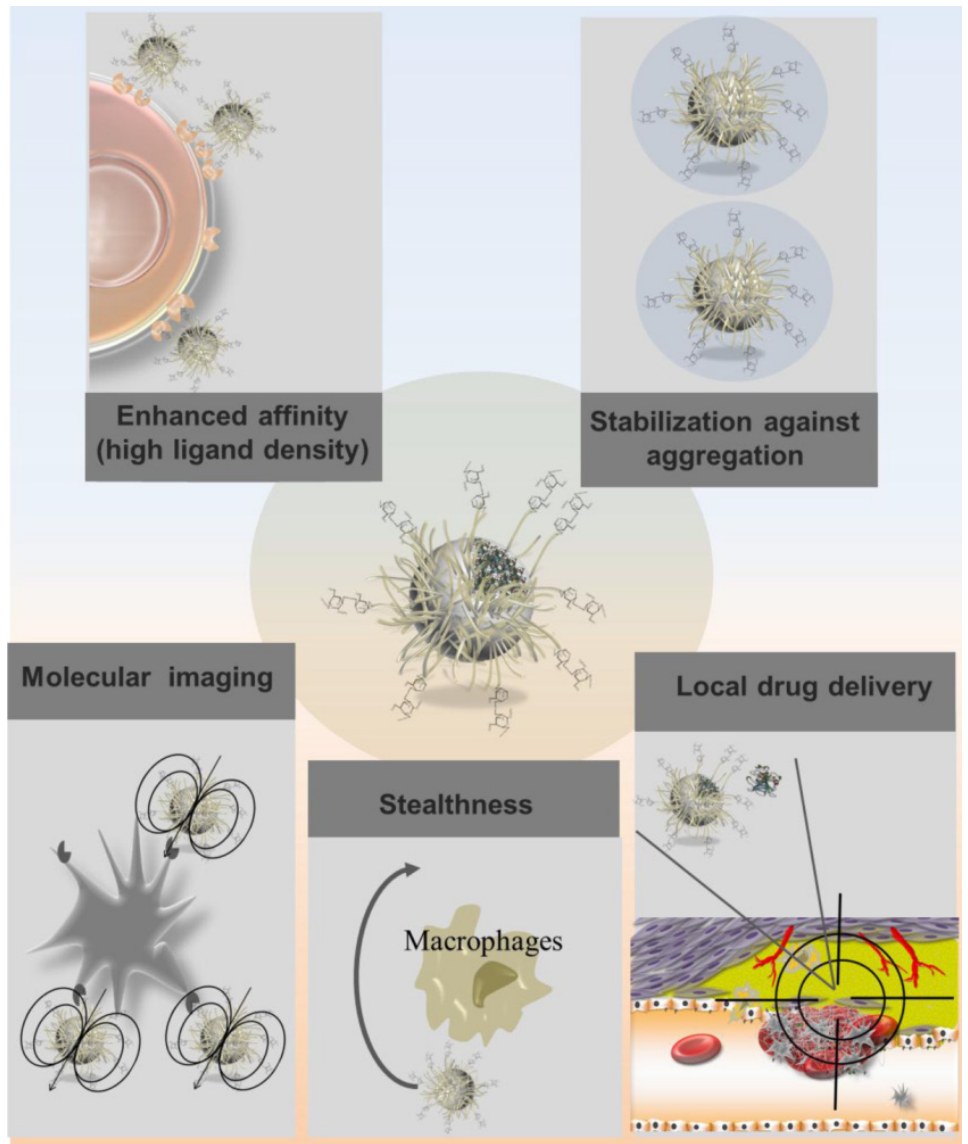

Figure 3: Advantages related to polysaccharide-based nanosystems considering their applications as drug carriers or imaging agents in atherothrombotic diseases.

The recognition properties conferred by polysaccharides provide nanoparticles with the ability to interact with important biomolecules involved in atherothrombotic disease such as P-selectins, cholesterol, anti-thrombin, as described in the previous subsection. This provides nanoparticles with high specificity for targeted drug delivery and molecular imaging in the management of cardiovascular diseases.

It is important to highlight that nanosystem capacity to accommodate a high density of ligands, due to their high surface area to volume ratios, actively promotes multivalent interactions with their binding partners (Fig. 3). This has significant implication in molecule recognition. In fact, ligand configuration directly influences presentation and cooperativity and thus impacts the interactions with the binding partners. Multiple epitopes of the same ligand exposed and a presentation in a three-dimensional format may result in enhanced affinities of the polysaccharide for its biological target.[7] As an example, Li and colleagues designed magnetic nanoparticles with multiple copies of cyclodextrin functionalizing the surface in order to enable simultaneous interaction with many cholesterol molecules, leading to high affinity binding with cholesterol for MRI detection purposes.[50] In fact, nanosystems may act as platform amplifying the weak affinities of polysaccharide ligands with biological molecules of interest. This highly contributes to an increased sensitivity for targeted drug delivery and molecular imaging in the management of cardiovascular diseases.

Besides conferring recognition, polysaccharides may also be used to avoid nanosystem uptake by the RES. In this regard, polysaccharides may act as hydrophilic layer sterically hindering opsonization and recognition by macrophages.[51] Alternatively, polysaccharide coating may prevent the opsonization of nanosystem directly inhibiting complement itself, as the case of heparin.[52] Stabilization of nanosystems is also achieved by polysaccharide coating.[53] Particles coated by a polymer shell prevent aggregation by reducing their surface energy in comparison with bare particles.[54] Polymer coating may also be performed for providing the nanosystem with a structure facilitating further chemical functionalization. This applies particularly to dextran-coated nanosystems, as discussed below. The main advantages of polysaccharide-based nanosystems are illustrated in Figure 3. The recognition properties conferred by polysaccha- 
rides enable nanosystems to interact with major players in atherothrombotic disease such as platelets, cholesterol, endothelial cells, macrophages, fibrin and thrombin. The key polysaccharide targets involved in atherothrombotic disease are thus depicted in Figure 4 .

\section{Production of polysaccharide-based to nanosystems}

A critical step in the preparation of polysaccharide-based nanosystems is the coupling method for attaching the polymer to the nanomaterial. Broadly speaking, the methods for producing polysaccharide-based nanosystems fall in the general categories of non-covalent and covalent approaches. Both approaches are associated with advantages and drawbacks, although covalent protocols are generally preferred due to the considerably higher stabilities. The method choice is based on nanosystem chemistry, desired particle size, polysaccharide chemistry and the intended application in order to afford efficient ligand coupling and to provide optimal ligand presentation.[7, 55, 56]

Non-covalent approaches rely on electrostatic interactions, hydrogen bonding and hydrophobic interactions. Although reversible nanosystem-polysaccharide association is obtained from such physical methods, there is the advantage of avoiding harsh preparation condition as well as toxic crosslinkers. $[1,57]$ For instance, dextran-coated quantum dots were synthesized via electrostatic interactions with negatively charged carboxymethyldextran.[58] In related approach, Huang and co-workers reported the production of silver/polysaccharide nanocomposites based on the interaction of the electron-rich at- oms of polar hydroxyl on polysaccharides and the electropositive transition metal cation.[59] Besides the fact that non-covalent approaches represent a green method providing an environmental friendly strategy to prepare polysaccharide-based nanoparticles, minimal or no chemical modification is required for the polysaccharide ligands and the nanomaterial substrates.[7, 60] Most of surface functionalization methods are covalent approaches. They are more advantageous considering the robust linkage and the stability of the surface ligand. Covalent attachment of polymers to nanosystems can be carried out by "grafting to" and "grafting from" methods. The "grafting to" method consists in using prefabricated polymers with reactive end groups to react with the functional groups on the surface of the nanosystem. The "grafting from" method involves reactive groups covalently attached to the nanosystem surfaces. The grafting reaction can proceed then by polymerization from the surface, initiated by reaction with monomers.[54, 61-63] The majority of methods to produce polysaccharide-coated nanosystems concern the "grafting to" method. Such direct polysaccharide conjugation to nanosystems is feasible when the ligand possesses a functional group that is reactive towards the nanosystem material. This is the case of polysaccharides presenting a thiol/disulfide group to be conjugated on metallic particles $(\mathrm{Au}, \mathrm{Ag}, \mathrm{Cu})$ and semiconductor ones (CdS, CdSe, $\mathrm{ZnS}$ ), and phosphates-containing ligands to be conjugated on metal oxide particles (iron oxide, $\mathrm{TiO}_{2}$ ). According to the nature of the substrate material, ligands possessing the corresponding functional groups are chosen.[57]

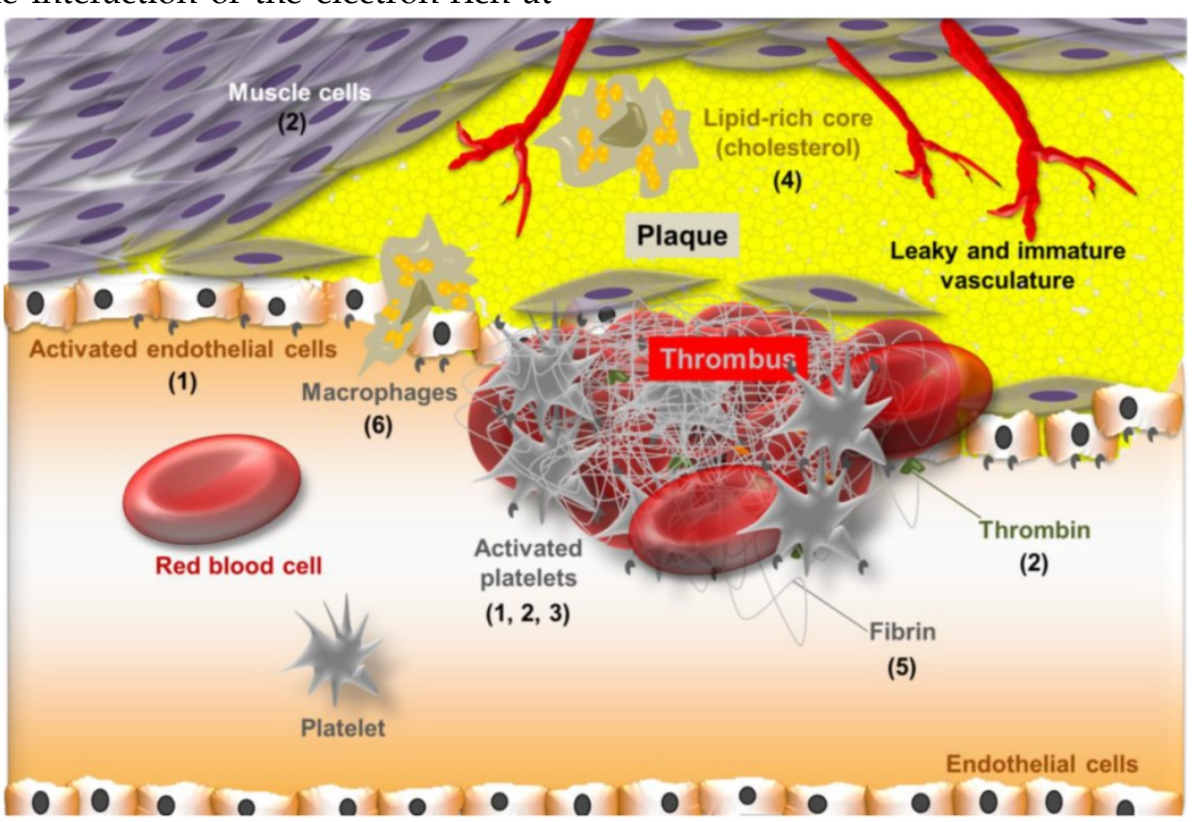

Figure 4: Schematic representation of atherothrombotic lesion highlighting some key features involved in polysaccharide recognition: sialyl Lewis ${ }^{\times}(I)$, heparin (2), fucoidan (3), cyclodextrin (4), chitosan (5) and hyaluronic acid (6). 
Concerning the characterization of polysaccharide-functionalized nanosystems, several techniques are available. Generally, zeta potential measurement is the most common technique used to establish the presence of a polysaccharidic coating. In the case of polysaccharide coating, the nanosystem charge may differ from particle previous to grafting.[43, 52] Colorimetric assays are frequently used enabling qualitative and quantitative analysis of functional groups. Grafting may be also analyzed via nuclear magnetic resonance, Fourier transform infrared spectroscopy, and surface-enhanced Raman spectroscopy, thermogravimetric analysis, elemental analysis and X-ray photoelectron spectroscopy. Additionally, by combining multi-angles laser light scattering, dynamic light scattering, microscopy techniques and small-angle X-ray scattering, physical characteristics of size, shape, and assembly behavior of the nanosystems can be elucidated.[7, 64-67]

\section{Polysaccharide-based nanosystems}

\section{Sialyl Lewis $x$}

The sialyl Lewis ${ }^{x}$ is a tetrasaccharide that contains fucose and sialic acid. It has been reported as a high affinity ligand for selectins,[68] which are adhesion molecules highly expressed by the dysfunctional endothelium and also by activated platelets in atherothrombotic process.[69-72] Selectins are composed of a lectin domain responsible for polysaccharide recognition. Among the lectin classes, the $\mathrm{C}$ type (C meaning calcium-requiring) represents a large class found in animals. These proteins present a common domain of 120 amino acids that is in charge of binding to sialyl Lewis ${ }^{x}$ or other polysaccharides. The calcium ion acts as a bridge between the protein and the polysaccharide through direct interactions with its hydroxyl groups. Additionally, two glutamate residues in the lectin bind to both the calcium ion and the polysaccharide, while other protein side chains interact with other hydroxyl groups on the polysaccharide forming hydrogen bonds.[73]

Sialyl Lewis ${ }^{x}$ has the unique ability to interact with the three family members of selectins, namely E, L, P.[68] This saccharidic structure has been conjugated to quantum dots in order to allow establishing the in vivo dynamic distribution profiles after intravenous injection in mice. In this study, interaction of functionalized quantum dots with selectin was not investigated, since the focus was the biodistribution in healthy mice. However, it was showed that quantum dots functionalized with sialyl Lewis $\mathrm{x}$ achieved a prolonged circulation lifetime of 2 hours without liver localization, differently from quantum dots functionalized with other glycans such as lactose.[74]

\section{Heparin}

Heparin is an unbranched acidic glycosaminoglycan rich in $\mathrm{N}$ - and $\mathrm{O}$-sulfate groups that is recognized by P-selectin and L-selectin.[24, 76] As indicated previously, heparin binds to antithrombin, which is an important player in coagulation process. Heparin binding relieves the natural repressed reactivity state of antithrombin transforming it into a fast inhibitor of blood clotting proteinases, which inactivates thrombin.[22] The configuration of $\mathrm{N}$ - and O-sulfate groups displayed by heparin is responsible for its high-affinity binding to antithrombin.[24]

Heparin-based nanoparticles were produced from amphiphilic copolymers of heparin and methyl methacrylate and in vivo blood circulation time of such nanoparticles was evaluated. A long-circulating time of more than 48 hours was obtained. Heparin succeeded in hiding the high opsonization effect associated to poly(methyl methacrylate) achieving a better stealthness.[77] Although the authors did not focus in demonstrating that the recognition properties of heparin was preserved after functionalization, these studies open perspectives of applications that are more than simply conferring hemo-compatibility and stealthness. As an example, Chauvierre et al. reported the production heparin-decorated poly(isobutyl cyanoacrylate) nanoparticles with reduced toxicity[51] and antithrombic activity,[52] additionally able to recognize typical heparin ligands such as von Willebrand factor.[78]

\section{Hyaluronic acid}

Hyaluronic acid is composed of D-glucuronic acid and N-acetyl-D-glucosamine units connected by $\beta-1,3-$, or $\beta-1,4-$ glycosidic bonds. Hyaluronic acid is a natural ligand of CD44 receptor expressed on macrophages. CD44 is a multifunctional receptor which is involved in cell-cell and cell-extracellular matrix interactions. Hyaluronic acid binds to CD44 close to its $\mathrm{NH}_{2}$ terminus which is situated within a 135-amino acid region of the receptor.[82-84] Therefore, hyaluronic acid enables atherosclerosis targeting via the recognition of receptors from macrophages, which are abundant in plaques. Hyaluronic acid-functionalized magnetic nanoparticles were reported to be labeled with a near-infrared fluorescence dye (Cy5.5) resulting in MR/optical dual imaging nanoprobes. However, no application related to atherothrombosis was exploited in this study.[86]

\section{Fucoidan}

Fucoidan is a branched polysaccharide sulfate ester with 1-fucose 4-sulfate building blocks that is recognized by P-selectin and L-selectin,[87] but not by E-selectin.[68] Fucoidan presents a high affinity to 
P-selectin. Indeed, low molecular weight fucoidan is most effective for binding to P-selectin when compared to other low molecular weight polysaccharides such as heparin and dextran sulfate.[88]

99mTc-fucoidan was designed for P-selectin molecular imaging by scintigraphy.[89] Alternatively, fucoidan was conjugated to ultra-small paramagnetic iron oxide nanoparticles via the functionalization of the carboxylmethyl dextran coating on nanoparticles in order to enable P-selectin molecular imaging by MRI.[90] This will be further discussed in the next section.

\section{Chitosan}

Chitosan is a linear polysaccharide composed of randomly distributed $\beta$-(1-4)-linked D-glucosamine and N-acetyl-D-glucosamine, which is obtained by the partial deacetylation of chitin. As a function of the $\mathrm{pH}$, amine groups from chitosan get protonated and become positively charged, making chitosan a water-soluble cationic polyelectrolyte.[91] Due to its charge, chitosan may interact with negatively charged molecules. Fibrin is a negatively-charged fibrous protein which has been reported to strongly interact electrostatically with chitosan via the interplay of the negative charges of carboxyl groups from amino acids such as glutamate of fibrin and the positive charges of amine groups of chitosan polymers.[92]

Chitosan electrostatic interaction with fibrin was tested for accelerating thrombolysis. Poly(lactic-coglycolic acid) nanoparticles loaded with tissue-plasminogen activator (t-PA) and coated with chitosan were designed. This will be further discussed in the next section.

\section{Cyclodextrin}

Cyclodextrins consist of a cyclic oligosaccharide formed by 6 to 8 a-1,4 linked D-glucopyranoside units (Fig. 1). Its external face is hydrophilic whereas the internal cavity provides a hydrophobic environment. This internal cavity has the ability to encapsulate hydrophobic molecules[93, 94] and it is responsible for cholesterol interaction.[95] In fact, the inner cavity of cyclodextrin provides a well-suited site for cholesterol accommodation. Once cholesterol enters the hydrophobic inner cavity of cyclodextrin, a stable host-guest inclusion complex is formed, which explains the affinity between these two molecules. Cholesterol is an important target in atherosclerosis process. Indeed, cholesterol accumulation is a hallmark of atherosclerosis disease.[96, 97] Cyclodextrins are known to avidly interact with cholesterol.[98] This property can be favorably exploited for cholesterol molecular imaging, as it will be further discussed in the next section."

\section{Dextran}

Dextran consists of a polysaccharide composed of glucose molecules connected in a 1-6 glucosidic linkage, in which side chains are connected in a 1-4 linkage. Dextran coating on nanoparticles and especially on iron oxide nanoparticles has been widely performed. On one side, dextran is used to prevent nanoparticles from aggregation. On the other side, dextran provides a framework for further chemical modifications in order to enable functionalization with target ligands.[101, 102] Particularly, a well-established platform consists of monocrystalline iron oxide nanoparticles presenting covalently cross-linked dextran know as cross-linked iron oxide nanoparticles, or CLIO. Such platform presents amine groups that are ready substrates for conjugation to targeting ligands.[103, 104] A discussion on the investigation of dextran-coated nanoparticles in the management of cardiovascular diseases is provided in the next section.

\section{Cardiovascular applications of polysac- charide-based nanoparticles}

The polysaccharide component of nanoparticles may play different roles in cardiovascular disease management. For instance, it may promote therapeutic effect, enable controlled release, endow recognition functions or provide a framework for further chemical modifications in order to enable functionalization with target ligands. Concerning the recognition feature, some polysaccharides seem to be more advantageous than others. As an example, hyaluronic acid recognition of macrophage receptor CD44 may have the disadvantage of providing a non-specific signal as macrophages are present in atheroma but also in off-target sites. Additionally, recognition properties mediated by some polysaccharides still need further investigation in order to enable molecular imaging in vivo. This is the case of $\beta$-cyclodextrin interaction with cholesterol. Notably, it has the disadvantage of relying only in a molecule accommodation process.[95] Other saccharide entities seem to be more promising for endowing nanoparticles with recognition properties for the management of cardiovascular diseases. This is the case, of Fucoidan[88] and Sialyl Lewis $\mathrm{x}$ [68] that interact with target selectins with high affinity. Such high affinity is quite advantageous for conferring targeting properties to nanoparticles. Examples related to the cardiovascular applications of polysaccharide-based nanoparticles are discussed in the following sub-sections.

\section{Therapy for intimal hyperplasia}

Heparin controlled release via polysaccharide-based nanoparticles was investigated for reduc- 
ing intimal hyperplasia. Heparin was associated to poly( $\varepsilon$-caprolactone) nanofibers. Fibers were electrospun from poly( $\varepsilon$-caprolactone) solutions containing heparin. A sustained heparin release was achieved over 14 days and the released heparin retained its biological activity. This system was effective in preventing the proliferation of vascular smooth muscle cell in vitro. This may indicate the potential of such fibers as candidates for heparin controlled delivery to a site of vascular injury.[80] In a different approach, heparin was encapsulated into poly(DL lactide-co-glycolide) (pLGA) spheres sequestered in an alginate gel. Heparin controlled release was achieved over a period of 25 days in vitro. Heparin-releasing gels were able to inhibit the proliferation of bovine vascular smooth muscle cells in tissue culture. Moreover, heparin controlled release from gels reduced intimal hyperplasia in in animal models of vascular disease.[44]

\section{Therapy for thrombosis}

Polysaccharide-based nanoparticles were investigated for fibrinolytic drug delivery in the treatment of thrombolysis. Poly(lactic-co-glycolic acid) nanoparticles loaded with tissue-plasminogen activator (t-PA) and coated with chitosan were designed for thrombolysis. The rationale of such delivery system for t-PA was based on chitosan electrostatic interaction with fibrin, as discussed in the previous section. Thrombolysis in a blood clot-occluded tube model was evaluated by determining clot lysis times and the masses of lysed clots. Chitosan-coated nanoparticles were able to penetrate the clot and markedly reduced t-PA clot lysis time in an in vitro model.[45] Permeation and clot dissolution patterns were also enhanced for poly(lactic-co-glycolic acid) nanoparticles loaded with t-PA and coated with chitosan, as compared to t-PA in solution.[13] Thrombolysis process using such nanoparticles could be enhanced by photomechanical drug delivery. In this approach, laser-induced hydrodynamic pressure elicit cavitation bubble expansion and collapse, resulting in damage in clot structure and increased binding sites available for t-PA.[21]

The iron oxide CLIO platform was employed for selective drug delivery. CLIO was functionalized with an activated factor XIII (FXIIIa)-sensitive peptide for targeting and recombinant t-PA. The fibrinolytic activity of the targeted nanoagent was demonstrated in vivo.[109] In an alternative approach, dextran-coated iron oxide nanoparticles were used for magnetically targeted thrombolytic therapy. Urokinase was conjugated to nanoparticles and local thrombolysis was achieved in vivo with an external magnetic field focused on the site of thrombus.[114]

\section{Therapy for atheroma}

The iron oxide nanoparticle platform CLIO, which is internalized by macrophages, was exploited to deliver drugs to macrophages in atheroma lesions. For this, CLIO was associated to the photosensitized drug m-THPC. Nanoparticle intravenous administration resulted in the localization within macrophage-rich atherosclerotic lesions. Macrophage uptake in atheroma lesions was attested by the detection of the fluorescent drug by intravital fluorescence microscopy. The atheroma was irradiated with $650 \mathrm{~nm}$ light in order to activate the drug to produce cytotoxic oxygen radicals. The photodynamic therapy performed resulted in eradication of inflammatory macrophages.[105]

\section{Imaging the activated endothelium or activated platelets}

Kasteren and colleagues made a step forward in the investigation of nanoparticles functionalized with sialyl Lewis ${ }^{x}$ to target the activated endothelium. They designed magnetic resonance imaging (MRI) detectable glyconanoparticles by conjugating the sialyl Lewis ${ }^{x}$ on a platform of cross-linked amine-functionalized iron oxide nanoparticles. They proposed glyconanoparticle application in vivo for detection of E-/P-selectin in a rat model of acute inflammation in the brain, in which the activated brain endothelium was induced by intracerebral microinjection of interleukin-1 $\beta$.[75]

Fucoidan-based systems were exploited for the molecular imaging of activated platelets. P-selectin binding by radiolabled ${ }^{99 \mathrm{~m} T c-f u c o i d a n}$ could be detected in vivo by scintigraphy in a rat model of platelet-rich arterial thrombi as well as in a myocardial ischemia-reperfusion model. The reported results demonstrated fucoidan potential as an efficient imaging agent in cardiovascular pathologies.[89] In a related study, fucoidan was conjugated to ultra-small paramagnetic iron oxide coated with carboxylmethyl dextran. Such fucoidan-decorated contrast agent for MRI succeeded in accurately detecting the thrombus in a rat model of an expanding aneurysm. There was a high correlation between MRI and histology of the regions corresponding to thrombus and P-selectin location, clearly indicating the effectiveness of such contrast agent in non-invasively thrombus detection. [90]

\section{Imaging for atherosclerosis}

Magnetic nanoparticles were functionalized with hyaluronic acid and the immobilized polysaccharide retained its specific biological recognition with the macrophage receptor CD44. The nanoparticles were successfully internalized by macrophages in vitro and 
the uptake was CD44-dependent. Nanoparticles were able to deliver a fluorescein cargo into cells and enabled detection by MRI. Although clinical application of these macrophage-targeted iron oxides has still to be demonstrated, such nanoparticles may be a potential platform for molecular imaging in atherosclerosis.[85]

Cholesterol molecular imaging by means of polysaccharide-based nanoparticles was investigated in vitro and ex vivo. $\beta$-cyclodextrin ability to interact with cholesterol was reported to be used for detection purposes via the fluorescent rhodamine $6 \mathrm{G}$ in vitro. For the design of such cholesterol detection system, graphene has chosen by virtue of its high surface area, high conductivity and strong fluorescence quenching property. The system principle is based on competitive host-guest interaction[99] between rhodamine 6G enclosed on $\beta$-cyclodextrin and cholesterol. When rhodamine $6 \mathrm{G}$ is in the inner compartment of $\beta$-cyclodextrin host, its fluorescence is quenched by graphene. However, rhodamine 6G fluorescence turns on after cholesterol replaces it. Thereby, cholesterol interaction translates into an optical signal.[100] In a different approach, cholesterol detection was performed by means of cyclodextrin-functionalized iron oxide nanoparticles. Selective binding to cholesterol crystals was demonstrated both in vitro and ex vivo using sections of atherosclerotic rabbit aorta tissues. By taking advantage of the magnetic properties of the iron oxide core, a proof of principle of cholesterol detection by MRI was provided. Although in vivo application of such cyclodextrin-functionalized nanoparticles has still to be demonstrated, they could be useful for non-invasive detection of cholesterol in atherosclerotic plaques.[50]

In a different approach, several ligands have been grafted to the iron oxide platform CLIO in order to achieve atherothrombosis target. CLIO grafting with VHPKQHR or VHSPNKK peptides targeted to vascular adhesion molecule-1 (VCAM-1) resulted in enhanced MR signal in aortic roots of apoE-/- mice enabling noninvasive imaging of VCAM-1-expressing endothelial cells and macrophages in atherosclerosis.[106, 107] CLIO platform was equally functionalized with peptide-targeting ligands with affinity to fibrin and activated factor XIII. In vivo and in vitro results confirmed selective targeting and detection by MRI.[108]

Apart from the CLIO platform, iron oxide nanoparticles coated with non-crosslinked dextran succeeded in selectively imaging plaque macrophages with high accuracy in vivo. This is the case of dextran-monocrystalline iron-oxide nanoparticles administrated intravenously in hyperlipidemic rabbits.[110, 111] An important example is the investiga- tion of superparamagnetic iron oxide nanoparticles stabilized with dextran and sodium citrate (Sinerem, Guerbet) for MRI detection of plaques of 11 symptomatic patients. The nanoparticles accumulated predominantly in macrophages in ruptured and rupture-prone human atherosclerotic plaques, indicating that it may be promising for differentiating low- and high-risk plaques. However, post-contrast imaging time needed to be long in order to allow uptake of iron particles by macrophages.[112] The same commercial nanoparticles were further investigated in an additional clinical trial confirming the potential of such nanoparticles to be used as a MRI contrast agent to identify inflamed atheromatous plaques. USPIO-enhanced MRI contrast took place between 24 and 48 hours after nanoparticle administration. [115]

\section{Conclusions and perspectives}

This article overviewed the role of polysaccharides as recognition moieties in atherothrombotic process in the basis of a structure-function relation. Advantages of polysaccharide association to nanosystems were evidenced. Recent accomplishments on polysaccharide-based nanosystems as drug delivery systems and targeted contrast agents for molecular imaging were outlined, including the current trend of merging both in a single entity for theranostics.

Polysaccharide-based nanosystems as drug delivery systems and targeted contrast agents for molecular imaging hold much promise in the management of atherothrombotic diseases. However, the field is still in its early days. Although very promising approaches have been designed, most of them are in the stage of pre-clinical studies or still relate to in vitro proofs of concept. Considerable challenges concern the optimization of nanoparticle properties in order to enhance targeting while minimizing non-specific tissue residence.

Although both molecular imaging and targeted therapeutics presented interesting proof of concept results, the ultimate performance of these nanosystems must be established in clinical trials. The clinical translation of these novel nanosystems is highly challenging, due to their inherent complexity. Besides, the choice of the nanoplatform, drug and imaging modalities is quite broad and should be carefully analyzed. The choice may be influenced by cost, availability, and the specific application to be considered. As it is the case for any novel pharmacological agent undergoing clinical trials, the investigation of polysaccharide-based nanoparticles will also require thorough evaluation for toxicity, pharmacokinetics and biodistribution.

Considering the studies discussed in the last 
section, clinical application of polysaccharide-based nanoparticles was limited to plaque macrophage imaging using dextran-coated iron oxides. To our knowledge, Sinerem (Guerbet) is the only commercial polysaccharide-based nanoparticle tested in clinical trials for managing atherothrombotic disease. However, Sinerem withdrawal was proposed in 2008 by the European Medicines Agency. Regarding such nanoparticles, it is important to consider safe iron doses. As a reference, iron dose should remain not higher than the one of USPIO $(2.6 \mathrm{mg}$ iron $/ \mathrm{kg}$ body weight) used for human oncological MRI.[116] Still considering the few clinical studies on plaque macrophage imaging using dextran-coated iron oxides, challenges of the approach are related to the intrinsic lower sensitivity of MRI, limiting the signal-to-noise ratio in high-resolution images required for coronary or carotid plaques. Future studies should focus on the translational potential of polysaccharide-based nanosystems enabling targeted therapy or imaging at the molecular level including multi-modal imaging. By taking profit from the advantageous properties of polysaccharides and nanosystems, such an approach may offer novel avenues in the management of atherothrombosis. Additionally, forthcoming advancements in imaging technology, including the development hybrid high performing scanners, combined to and the future improvement of nanocarrier platforms may open bright perspectives for polysaccharide-based nanosystems. Meanwhile, the investigation of polysaccharide-based nanosystems in animal models as molecular imaging tools and targetable drug delivery systems holds great promise in extending current knowledge of therapy/imaging limits as well as of the pathological mechanisms involved in atherothrombotic diseases.

\section{Acknowledgements}

The authors acknowledge NanoAthero FP-7 project (Grant agreement N³09820) for funding.

\section{Competing Interests}

The authors have declared that no competing interest exists.

\section{References}

1. Nitta SK, Numata K. Biopolymer-Based Nanoparticles for Drug/Gene Delivery and Tissue Engineering. Int. J. Mol. Sci. 2013; 14: 1629-54.

2. Oh JK, Lee DI, Park JM. Biopolymer-based microgels/nanogels for drug delivery applications. Prog. Polym. Sci. 2009; 34: 1261-82.

3. Muthana SM, Campbell CT, Gildersleeve JC. Modifications of Glycans: Biological Significance and Therapeutic Opportunities. ACS Chem. Biol. 2012; 7: 31-43.

4. Sinha V, Kumria R. Polysaccharides in colon-specific drug delivery. Int. J. Pharm. 2001; 224: 19-38.

5. Quignard F, Di Renzo F, Guibal E. From natural polysaccharides to materials for catalysis, adsorption, and remediation. Top. Curr. Chem. 2010; 294: 165-97.

6. Raman R, Sasisekharan V, Sasisekharan R.. Structural Insights into Biological Roles of Protein-Glycosaminoglycan Interactions. Chem. Biol. 2005; 12: 267-77.
7. Wang $X$, Ramström $O$, Yan M. Glyconanomaterials: synthesis, characterization, and ligand presentation. Adv. Mater. 2010; 22: 1946-53.

8. Lu D, Xiao C, Xu S. Starch-based completely biodegradable polymer materials. Express Polym. Lett. 2009; 3: 366-75.

9. Gunatillake PA, Adhikari R. Biodegradable synthetic polymers for tissue engineering. Eur. Cell Mater. 2003; 5: 1-16.

10. El-Boubbou K, Huang X. Glyco-Nanomaterials: Translating Insights from the Sugar-Code to Biomedical Applications. Curr. Med. Chem. 2011; 18: 2060-78.

11. Varki A. Biological roles of oligosaccharides: all of the theories are correct. Glycobiology. 1993; 3: 97-130.

12. Varki A. Selectin ligands. Proc. Natl. Acad. Sci. U.S.A. . 1994; 91: 7390-7.

13. Pochechueva T, Galanina O, Ushakova N, Preobrazhenskaya M, Sablina M, Nifantiev N, et al. Uncharged P-selectin blockers. Glycoconjugate J. 2004; 20: 91-7.

14. Finlay BB, Cossart P. Exploitation of mammalian host cell functions by bacterial pathogens. Science. 1997; 276: 718-25.

15. Weiner R, Langille S, Quintero E. Structure, function and immunochemistry of bacterial exopolysaccharides. J. Ind. Microbiol. 1995; 15: 339-46.

16. Bourguignon LYW, Zhu H, Shao L, Chen YW. CD44 Interaction with Tiam1 Promotes Rac1 Signaling and Hyaluronic Acid-mediated Breast Tumor Cell Migration. J. Biol. Chem. 2000; 275: 1829-38.

17. Tzianabos AO. Polysaccharide Immunomodulators as Therapeutic Agents: Structural Aspects and Biologic Function. Clin. Microbiol. Rev. 2000; 13: 523-33.

18. Wong K-H, Lai CKM, Cheung PCK. Immunomodulatory activities of mushroom sclerotial polysaccharides. Food Hydrocolloids. 2011; 25: 150-8.

19. Xiang L, Sze CS, Ng T, Tong Y, Shaw P, Tang CS, et al. Polysaccharides of Dendrobium officinale inhibit TNF-a-induced apoptosis in A-253 cell line. Inflammation Res. 2013; 62: 313-24.

20. Müller A, Rice PJ, Ensley HE, Coogan PS, Kalbfleish JH, Kelley JL, et al. Receptor binding and internalization of a water-soluble (1-->3)-beta-D-glucan biologic response modifier in two monocyte/macrophage cell lines. J. Immunol. 1996; 156: 3418-25.

21. Medeiros SDV, Cordeiro SL, Cavalcanti JEC, Melchuna KM, Lima AMdS, Medeiros AC, et al. Effects of Purified Saccharomyces cerevisiae $(1 \rightarrow$ 3)- $\beta$-Glucan on Venous Ulcer Healing. Int. J. Mol. Sci. 2012; 13: 8142-58.

22. Olson ST, Richard B, Izaguirre G, Schedin-Weiss S, Gettins PG. Molecular mechanisms of antithrombin-heparin regulation of blood clotting proteinases. A paradigm for understanding proteinase regulation by serpin family protein proteinase inhibitors. Biochimie. 2010; 92: 1587-96.

23. Tovar AM, Capillé NV, Santos GR, Vairo BC, Oliveira S-N, Fonseca RJ, et al. Heparin from bovine intestinal mucosa: Glycans with multiple sulfation patterns and anticoagulant effects. Thromb. Haemost. 2012; 107: 903-15.

24. Nelson RM, Cecconi O, Roberts WG, Aruffo A, Linhardt RJ, Bevilacqua MP. Heparin oligosaccharides bind L-and P-selectin and inhibit acute inflammation. Blood. 1993; 82: 3253-8.

25. Bourin M-C, Lindahl U. Glycosaminoglycans and the regulation of blood coagulation. Biochem. J. 1993; 289: 313.

26. Letourneur D, Caleb BL, Castellot JJ. Heparin binding, internalization, and metabolism in vascular smooth muscle cells: I. Upregulation of heparin binding correlates with antiproliferative activity. J Cell Physiol. 1995; 165: 676-86.

27. Furie B, Furie BC. Mechanisms of thrombus formation. N. Engl. J. Med. 2008; 359: 938-49.

28. Roger VL, Go AS, Lloyd-Jones DM, Adams RJ, Berry JD, Brown TM, et al. Heart Disease and Stroke Statistics-2011 Update: a report from the American Heart Association. Circulation. 2011; 123: e18-e209.

29. Ross R. Atherosclerosis - an inflammatory disease. N. Engl. J. Med. 1999; 340: $115-26$.

30. Abi-Younes S, Sauty A, Mach F, Sukhova GK, Libby P, Luster AD. The Stromal Cell-Derived Factor-1 Chemokine Is a Potent Platelet Agonist Highly Expressed in Atherosclerotic Plaques. Circ. Res. 2000; 86: 131-8.

31. Vancraeynest D, Pasquet A, Roelants V, Gerber BL, Vanoverschelde J-LJ. Imaging the Vulnerable Plaque. J. Am. Coll. Cardiol. 2011; 57: 1961-79.

32. Klink A, Hyafil F, Rudd J, Faries P, Fuster V, Mallat Z, et al. Diagnostic and therapeutic strategies for small abdominal aortic aneurysms. Nat. Rev. Cardiol. 2011; 8: 338-47.

33. Hansson GK. Inflammation, atherosclerosis, and coronary artery disease. N. Engl. J. Med. 2005; 352: 1685-95.

34. Michelson AD. Antiplatelet therapies for the treatment of cardiovascular disease. Nat. Rev. Drug Discovery. 2010; 9: 154-69.

35. Kim BY, Rutka JT, Chan WC. Nanomedicine. N. Engl. J. Med. 2010; 363: 2434-43.

36. Mailänder V, Landfester K. Interaction of Nanoparticles with Cells. Biomacromolecules. 2009; 10: 2379-400.

37. Lewis DR, Kamisoglu K, York AW, Moghe PV. Polymer-based therapeutics: nanoassemblies and nanoparticles for management of atherosclerosis. Wiley Interdiscip. Rev. Nanomed. Nanobiotechnol. 2011; 3: 400-20.

38. Maeda $\mathrm{H}$. The enhanced permeability and retention (EPR) effect in tumor vasculature: the key role of tumor-selective macromolecular drug targeting. Adv. Enzyme Regul. 2001; 41: 189-207.

39. Greish K. Enhanced permeability and retention (EPR) effect for anticancer nanomedicine drug targeting. Methods Mol. Biol. 2010; 624: 25-37.

40. Doyle B, Caplice N. Plaque Neovascularization and Antiangiogenic Therapy for Atherosclerosis. J. Am. Coll. Cardiol. 2007; 49: 2073-80. 
41. Liu Z, Jiao Y, Wang Y, Zhou C, Zhang Z. Polysaccharides-based nanoparticles as drug delivery systems. Adv. Drug Delivery Rev. 2008; 60: 1650-62.

42. Chacko A-M, Hood ED, Zern BJ, Muzykantov VR. Targeted nanocarriers for imaging and therapy of vascular inflammation. Curr. Opin. Colloid Interface Sci. 2011; 16: 215-27.

43. Lemarchand C, Gref R, Couvreur P. Polysaccharide-decorated nanoparticles. Eur. J. Pharm. Biopharm. 2004; 58: 327-41.

44. Edelmana ER, Nathan A, Katada M, Gates J, Karnovsky MJ. Perivascular graft heparin delivery using biodegradable polymer wraps. Biomaterials. 2000; 21: 2279-86.

45. Chung T-W, Wang S-S, Tsai W-J. Accelerating thrombolysis with chitosan-coated plasminogen activators encapsulated in poly-(lactide-co-glycolide) (PLGA) nanoparticles. Biomaterials. 2008; 29: 228-37.

46. Michalet X, Pinaud F, Bentolila L, Tsay J, Doose S, Li J, et al. Quantum dots for live cells, in vivo imaging, and diagnostics. Science. 2005; 307: 538-44

47. de Montferrand C, Lalatonne $\mathrm{Y}$, Bonnin D, Lièvre N, Lecouvey M, Monod P, et al. Size-Dependent Nonlinear Weak-Field Magnetic Behavior of Maghemite Nanoparticles. Small. 2012; 8: 1945-56.

48. Moghimi SM, Szebeni J. Stealth liposomes and long circulating nanoparticles: critical issues in pharmacokinetics, opsonization and protein-binding properties. Prog. Lipid Res. 2003; 42: 463-78.

49. Chellat F, Merhi Y, Moreau A, Yahia LH. Therapeutic potential of nanoparticulate systems for macrophage targeting. Biomaterials. 2005; 26: 7260-75.

50. Li H, El-Dakdouki MH, Zhu DC, Abela GS, Huang X. Synthesis of $\beta$-cyclodextrin conjugated superparamagnetic iron oxide nanoparticles for selective binding and detection of cholesterol crystals. Chem. Commun. 2012; 48: 3385-7.

51. Chauvierre C, Leclerc L, Labarre D, Appel M, Marden MC, Couvreur P, et al. Enhancing the tolerance of poly (isobutylcyanoacrylate) nanoparticles with a modular surface design. Int. J. Pharm. 2007; 338: 327-32.

52. Chauvierre C, Labarre D, Couvreur P, Vauthier C. Novel polysaccharide-decorated poly(isobutyl cyanoacrylate) nanoparticles. Pharm. Res. 2003; 20: 1786-93.

53. Shenhar R, Norsten TB, Rotello VM. Polymer-Mediated Nanoparticle Assembly: Structural Control and Applications. Adv. Mater. 2005; 17: 657-69.

54. Rozenberg B, Tenne R. Polymer-assisted fabrication of nanoparticles and nanocomposites. Prog. Polym. Sci. 2008; 33: 40-112.

55. Balazs AC, Emrick T, Russell TP. Nanoparticle polymer composites: Where two small worlds meet. Science. 2006; 314: 1107-10.

56. Rao JP, Geckeler KE. Polymer nanoparticles: Preparation techniques and size-control parameters. Prog. Polym. Sci. 2011; 36: 887-913

57. Wang X, Liu L-H, Ramström O, Yan M. Engineering nanomaterial surfaces for biomedical applications. Exp. Biol. Med. 2009; 234: 1128-39.

58. Chen Y, Ji T, Rosenzweig Z. Synthesis of glyconanospheres containing luminescent CdSe-ZnS quantum dots. Nano Lett. 2003; 3: 581-4.

59. Huang H, Yuan $Q$, Yang X. Preparation and characterization of metal-chitosan nanocomposites. Colloids Surf B Biointerfaces.. 2004; 39: 31-7.

60. de la Fuente JM, Penadés S. Glyconanoparticles: Types, synthesis and applications in glycoscience, biomedicine and material science. Biochim. Biophys. Acta, Gen. Subj. 2006; 1760: 636-51.

61. Qin S, Qin D, Ford WT, Resasco DE, Herrera JE. Functionalization of single-walled carbon nanotubes with polystyrene via grafting to and grafting from methods. Macromolecules. 2004; 37: 752-7.

62. Berger S, Synytska A, Ionov L, Eichhorn K-J, Stamm M. Stimuli-responsive bicomponent polymer janus particles by "grafting from"/"grafting to" approaches. Macromolecules. 2008; 41: 9669-76.

63. Minko S. Grafting on solid surfaces: "Grafting to" and "grafting from" methods. Berlin, Heidelberg: Springer. 2008

64. Sperling R, Parak W. Surface modification, functionalization and bioconjugation of colloidal inorganic nanoparticles. Phil. Trans. R. Soc. A. 2010; 368: 1333-83.

65. Cliffel DE, Turner BN, Huffman BJ. Nanoparticle-based biologic mimetics. Wiley Interdiscip. Rev. Nanomed. Nanobiotechnol. 2008; 1: 47-59.

66. Silva A, Silva E, Carrico AT, Egito E. Magnetic carriers: a promising device for targeting drugs into the human body. Curr. Pharm. Des. 2007; 13: 1179-85.

67. Brun-Graeppi AK, Richard C, Bessodes M, Scherman D, Merten O-W. Thermoresponsive surfaces for cell culture and enzyme-free cell detachment. Prog. Polym. Sci. 2010; 35: 1311-24.

68. Foxall C, Watson SR, Dowbenko D, Fennie C, Lasky LA, Kiso M, et al. The three members of the selectin receptor family recognize a common carbohydrate epitope, the sialyl Lewis (x) oligosaccharide. J. Cell Biol. 1992; 117: 895-902.

69. Kaplan ZS, Jackson SP. The Role of Platelets in Atherothrombosis. Hematology. 2011; 2011: 51-61.

70. te Boekhorst BC, van Tilborg GA, Strijkers GJ, Nicolay K. Molecular MRI of inflammation in atherosclerosis. Curr. Cardiovasc. Imaging Rep. 2012; 5: 1-9.

71. Lobatto ME, Fuster V, Fayad ZA, Mulder WJ. Perspectives and opportunities for nanomedicine in the management of atherosclerosis. Nat. Rev. Drug Discovery. 2011; 10: 835-52

72. Falk E. Pathogenesis of atherosclerosis. J. Am. Coll. Cardiol. 2006; 47: C7-C12

73. Berg JM, Tymoczko JL, Stryer L. Lectins Are Specific Carbohydrate-Binding Proteins. In: Biochemistry. W H Freeman. 1999.
74. Ohyanagi T, Nagahori N, Shimawaki K, Hinou H, Yamashita T, Sasaki A, et al. Importance of Sialic Acid Residues Illuminated by Live Animal Imaging Using Phosphorylcholine Self-Assembled Monolayer-Coated Quantum Dots. J. Am. Chem. Soc. 2011; 133: 12507-17.

75. Van Kasteren SI, Campbell SJ, Serres S, Anthony DC, Sibson NR, Davis BG. Glyconanoparticles allow pre-symptomatic in vivo imaging of brain disease. Proc. Natl. Acad. Sci. U.S.A. . 2009; 106: 18-23.

76. Bevilacqua MP, Nelson RM. Selectins. J. Clin. Invest. 1993; 91: 379-87.

77. Passirani C, Barratt G, Devissaguet J-P, Labarre D. Long-circulating nanopartides bearing heparin or dextran covalently bound to poly (methyl methacrylate). Pharm. Res. 1998; 15: 1046-50.

78. Chauvierre C, Marden MC, Vauthier C, Labarre D, Couvreur P, Leclerc L. Heparin coated poly (alkylcyanoacrylate) nanoparticles coupled to hemoglobin: a new oxygen carrier. Biomaterials. 2004; 25: 3081-6.

79. Wang K-J, Li H-X, Song Y-M, Luan N-N, Xian P. Study on syntheses and anticoagulant action of heparin/rare earth nano-oxides hybrid material. Biopolymers. 2010; 93: 887-92.

80. Luong-Van E, Grøndahl L, Chua KN, Leong KW, Nurcombe V, Cool SM. Controlled release of heparin from poly( $\varepsilon$-caprolactone) electrospun fibers. Biomaterials. 2006; 27: 2042-50.

81. Kwon IK Matsuda T. Co-Electrospun Nanofiber Fabrics of Poly(l-lactide-co- $\varepsilon$-caprolactone) with Type I Collagen or Heparin. Biomacromolecules. 2005; 6: 2096-105.

82. Peach RJ, Hollenbaugh D, Stamenkovic I, Aruffo A. Identification of hyaluronic acid binding sites in the extracellular domain of CD44. J. Cell Biol. 1993; 122: 257-64

83. Naor D, Sionov RV, Ish-Shalom D. CD44: structure, function and association with the malignant process. Adv. Cancer Res. 1997; 71: 241-319.

84. Ponta H, Sherman L, Herrlich PA. CD44: From adhesion molecules to signalling regulators. Nat. Rev. Mol. Cell Biol. 2003; 4: 33-45.

85. Kamat M, El-Boubbou K, Zhu DC, Lansdell T, Lu X, Li W, et al. Hyaluronic Acid Immobilized Magnetic Nanoparticles for Active Targeting and Imaging of Macrophages. Bioconjugate Chem. 2010; 21: 2128-35.

86. Lee D-E, Kim AY, Saravanakumar G, Koo H, Kwon IC, Choi K, et al. Hyaluronidase-sensitive SPIONs for MR/optical dual imaging nanoprobes. Macromol. Res. 2011; 19: 861-7.

87. H. Thorlacius, B. Vollmar, U.T. Seyfert, D. Vestweber, M.D. Menger. The polysaccharide fucoidan inhibits microvascular thrombus formation independently from P- and L-selectin function in vivo. Eur. J. Clin. Invest. 2000; 30: 804-10.

88. Bachelet L, Bertholon I, Lavigne D, Vassy R, Jandrot-Perrus M, Chaubet F, et al. Affinity of low molecular weight fucoidan for P-selectin triggers its binding to activated human platelets. Biochim. Biophys. Acta, Gen. Subj. 2009; 1790: $141-6$

89. Rouzet F, Bachelet-Violette L, Alsac J-M, Suzuki M, Meulemans A, Louedec L, et al. Radiolabeled fucoidan as a P-selectin targeting agent for in vivo imaging of platelet-rich thrombus and endothelial activation. J. Nucl. Med. 2011; 52: $1433-40$

90. Suzuki M, Serfaty J-M, Bachelet L, Beilvert A, Louedec L, Chaubet F, et al. In vivo targeted molecular imaging for activated platelets by mri using USPIO-fucoidan in rat abdominal aortic aneuryms model. J. Cardiovasc. Magn. Reson. 2011; 13: 372

91. Dash M, Chiellini F, Ottenbrite R, Chiellini E. Chitosan-A versatile semi-synthetic polymer in biomedical applications. Prog. Polym. Sci. 2011; 36: 981-1014.

92. Chung T-W, Yang M-C, Tsai W-J. A fibrin encapsulated liposomes-in-chitosan matrix (FLCM) for delivering water-soluble drugs: Influences of the surface properties of liposomes and the crosslinked fibrin network. Int. J. Pharm. 2006; 311: $122-9$

93. Xiong L, Zheng L, Han K, Liu Q, Li Y, Liu W, et al. Drug carriers based on cyclodextrin inclusion complexes for the controlled release of hydrophobic drugs. J. Controlled Release. 2011; 152: e121-3.

94. $\mathrm{He} \mathrm{Q}, \mathrm{Wu} \mathrm{W}$, Xiu K, Zhang Q, Xu F, Li J. Controlled drug release system based on cyclodextrin-conjugated poly (lactic acid)-b-poly (ethylene glycol) micelles. Int. J. Pharm. 2013; 443: 110-9.

95. Christian A, Haynes M, Phillips M, Rothblat G. Use of cyclodextrins for manipulating cellular cholesterol content. J. Lipid Res. 1997; 38: 2264-72.

96. Michel JB, Virmani R, Arbustini E, Pasterkamp G. Intraplaque haemorrhages as the trigger of plaque vulnerability. Eur. Heart J. 2011; 32: 1977-85.

97. Meyrier A. Cholesterol crystal embolism: Diagnosis and treatment. Kidney Int. 2006; 69: 1308-12.

98. Yancey PG, Rodrigueza WV, Kilsdonk EP, Stoudt GW, Johnson WI, Phillips MC, et al. Cellular cholesterol efflux mediated by cyclodextrins. J. Biol. Chem. 1996; 271: 16026-34.

99. Das S, Joseph MT, Sarkar D. Hydrogen Bonding Interpolymer Complex Formation and Study of Its Host-Guest Interaction with Cyclodextrin and Its Application as an Active Delivery Vehicle. Langmuir. 2013; 29: 1818-30.

100. Mandal A, Jana NR. Fluorescent detection of cholesterol using $\beta$-cyclodextrin functionalized graphene. Chem. Commun. 2012; 48: 7316-8

101. Mornet S, Portier J, Duguet E. A method for synthesis and functionalization of ultrasmall superparamagnetic covalent carriers based on maghemite and dextran. J. Magn. Magn. Mater. 2005; 293: 127-34.

102. Berry CC, Curtis AS. Functionalisation of magnetic nanoparticles for applications in biomedicine. J. Phys. D: Appl. Phys. 2003; 36: R198-206. 
103. McCarthy JR, Weissleder R. Multifunctional magnetic nanoparticles for targeted imaging and therapy. Adv. Drug Deliv. Rev. 2008; 60: 1241-51.

104. Tassa C, Shaw SY, Weissleder R. Dextran-coated iron oxide nanoparticles: a versatile platform for targeted molecular imaging, molecular diagnostics, and therapy. Acc. Chem. Res. 2011; 44: 842-52.

105. McCarthy JR, Korngold E, Weissleder R, Jaffer FA. A Light-Activated Theranostic Nanoagent for Targeted Macrophage Ablation in Inflammatory Atherosclerosis. Small. 2010; 6: 2041-9.

106. Nahrendorf M, Jaffer FA, Kelly KA, Sosnovik DE, Aikawa E, Libby P, et al. Noninvasive Vascular Cell Adhesion Molecule-1 Imaging Identifies Inflammatory Activation of Cells in Atherosclerosis. Circulation. 2006; 114: 1504-11.

107. Kelly KA, Allport JR, Tsourkas A, Shinde-Patil VR, Josephson L, Weissleder R. Detection of Vascular Adhesion Molecule-1 Expression Using a Novel Multimodal Nanoparticle. Circ. Res. 2005; 96: 327-36.

108. McCarthy JR, Patel P, Botnaru I, Haghayeghi P, Weissleder R, Jaffer FA. Multimodal Nanoagents for the Detection of Intravascular Thrombi. Bioconjugate Chem. 2009; 20: 1251-5.

109. McCarthy JR, Sazonova IY, Erdem SS, Hara T, Thompson BD, Patel P, et al. Multifunctional nanoagent for thrombus-targeted fibrinolytic therapy. Nanomedicine. 2012; 7: 1017-28

110. Korosoglou G, Weiss RG, Kedziorek DA, Walczak P, Gilson WD, Schär M, et al. Noninvasive detection of macrophage-rich atherosclerotic plaque in hyperlipidemic rabbits using "positive contrast" magnetic resonance imaging. J. Am. Coll. Cardiol. 2008; 52: 483-91.

111. Hyafil F, Laissy J-P, Mazighi M, Tchétché D, Louedec L, Adle-Biassette H, et al. Ferumoxtran-10-Enhanced MRI of the Hypercholesterolemic Rabbit Aorta Relationship Between Signal Loss and Macrophage Infiltration. Arterioscler. Thromb. Vasc. Biol. 2006; 26: 176-81.

112. Kooi ME, Cappendijk VC, Cleutjens KBJM, Kessels AGH, Kitslaar PJEHM, Borgers M, et al. Accumulation of Ultrasmall Superparamagnetic Particles of Iron Oxide in Human Atherosclerotic Plaques Can Be Detected by In Vivo Magnetic Resonance Imaging. Circulation. 2003; 107: 2453-8.

113. Tietze R, Lyer S, Dürr S, Alexiou C. Nanoparticles for cancer therapy using magnetic forces. Nanomedicine. 2012; 7: 447-57.

114. Bi F, Zhang J, Su Y, Tang Y-C, Liu J-N. Chemical conjugation of urokinase to magnetic nanoparticles for targeted thrombolysis. Biomaterials. 2009; 30: 5125-30.

115. Trivedi RA, U-King-Im J-M, Graves MJ, Cross JJ, Horsley J, Goddard MJ, et al. In Vivo Detection of Macrophages in Human Carotid Atheroma: Temporal Dependence of Ultrasmall Superparamagnetic Particles of Iron Oxide-Enhanced MRI. Stroke. 2004; 35: 1631-5.

116. Will O, Purkayastha S, Chan C, Athanasiou T, Darzi AW, Gedroyc W, et al. Diagnostic precision of nanoparticle-enhanced MRI for lymph-node metastases: a meta-analysis. Lancet Oncol. 2006; 7: 52-60. 